\title{
Buchbesprechung: Trotzdem von Ferdinand von Schirach und Alexander Kluge
}

Thorsten Päplow

University of Agder

Deutschland im Frühjahr 2020, die Corona-Infektionslage wird zunehmend kritischer, die Verunsicherung unter den Menschen ist groß, und die Politik ringt mit Entscheidungen. Vor diesem Hintergrund führen der Schriftsteller und Jurist Ferdinand von Schirach und der Jurist, Filmemacher und Schriftsteller Alexander Kluge am 30. März 2020 per Instant-MessangerDienst ein Gespräch in zwei Teilen. Diese wurden im ca. 70-seitigen Bändchen Trotzdem veröffentlicht, das es aus dem Stand und über Monate hinweg in die Spitze der BestsellerListen für Sachbücher schaffte. Grund genug, dieses Buch zu besprechen, denn obwohl Trotzdem mit einigen Befremdlichkeiten aufwartet, hat es in Deutschland offenbar einen Nerv getroffen. Doch welchen?

Dies ist natürlich nicht ganz einfach zu sagen. Schaut man sich die vielfältigen Kommentare von Lesern auf den Webseiten bekannter Online-Händler an, sticht eines besonders hervor. Die Leser erwarten augenscheinlich andere Fragen und Antworten, als sie von Politikern, Epidemiologen oder anderen Wissenschaftlern zur Corona-Situation gestellt bzw. gegeben werden. Ob diese Erwartung von Trotzdem dann auch erfüllt wird, scheint für das jeweilige Urteil über dieses Buch wesentlich zu sein.

Damit seien die Bemühungen von Politikern, Experten und Wissenschaftlern in Nachrichten- und Diskussionssendungen, in Zeitungen und Podcasts usw. keineswegs geschmälert. Doch der Verkaufserfolg dieses Bändchens scheint von einem Bedürfnis nach einer anderen, vielleicht grundlegenderen Perspektive zu zeugen. Denn welche CoronaAussage aus dem März 2020 könnte noch Monate später auf solch ein Interesse stoßen, während sich die alltägliche Situation der Menschen und auch die Wissenslage seitdem kontinuierlich und zum Teil stark verändern?

Doch zunächst zu einigen der oben erwähnten Befremdlichkeiten. Ganz im Zeichen der Abstandswahrung, so lässt sich vermuten, findet dieses ,Gespräch` digital statt und ist unterteilt in den Vormittag und den Nachmittag des 30. März 2020. Warum von Schirach (in 


\section{Nordic Journal of Modern Language Methodology}

Berlin) und Kluge (in München) einen Instant-Messanger-Dienst und nicht eine Telefon- oder Videokonferenz gewählt haben, die sich im Nachhinein ja auch vertextlichen ließe, wird nicht klar. Vielleicht weil sich beide, als Juristen und Schriftsteller, in erster Linie als Männer des geschriebenen Wortes verstehen. Was auch immer der Grund sein mag, so führt dieses Format doch zu einem ungewöhnlichen Duktus, der teilweise etwas hölzern daherkommt. Dies wird verstärkt von dem Umstand, den man aus anderen Formaten kennt, nämlich wenn ein Experte einem anderen Experten aus demselben oder einem benachbarten Fach Fragen stellt, deren Beantwortung er auch selbst übernehmen könnte. So wirkt etwa der Austausch zur Verfassungskonformität der Einschränkung des Demonstrationsrechtes vor dem Hintergrund der Corona-Infektionslage etwas erzwungen, wenn man bedenkt, dass sich hier zwei Juristen miteinander unterhalten:

\section{KLUGE}

Erlaubt unsere Verfassung das?

\section{SCHIRACH}

Vereinfacht gesagt muss jede Maßnahme, die Grundrechte einschränkt, verhältnismäßig sein. Das ist sie, wenn sie vier Voraussetzungen erfüllt: Sie muss einen legitimen Zweck verfolgen, geeignet, erforderlich und angemessen sein.

\section{KLUGE}

Können Sie das ein bisschen näher erläutern?

\section{SCHIRACH}

Der Zweck, den die Maßnahme verfolgt, muss legitim sein. Sie muss also mit den Werten des Grundgesetzes übereinstimmen.

\section{KLUGE}

Das Leben von Menschen zu retten ist sicher ein legitimer Zweck.

\section{SCHIRACH}




\section{Nordic Journal of Modern Language Methodology}

Natürlich. Aber jedes Jahr sterben Tausende bei Autounfällen. Das will ein Politiker verhindern. Er schlägt deshalb vor, allen Menschen die Fahrerlaubnis zu entziehen. Sein Ziel - das Leben von Menschen zu schützen - ist legitim.

\section{KLUGE}

Aber es ist in diesem Fall nicht angemessen.

\section{SCHIRACH}

Ja.

(von Schirach \& Kluge, 2020, S. 14-15)

Wen dieses Format stört, bei dem sich zwei ältere gebildete Männer nach diesem Muster die Lieblingsbälle zuspielen, dem sei von der Lektüre dieses Bändchens abgeraten. Wer sich jedoch darauf einlassen kann, verfolgt eine auch für Laien nachvollziehbare,Diskussion“ darüber, wie der Wortlaut des Grundgesetzes ausgelegt werden kann und muss, um in besonderen Fällen oder Notlagen Grundrechte verfassungskonform einzuschränken. Auch zeichnen von Schirach und Kluge die allmähliche Entwicklung der Grund- und Menschenrechte vom Gang von Canossa über Locke, Montesquieu und die amerikanische Bill of Rights nach. Dies geschieht teilweise auf eine Art und Weise, dass man sich als Leser ab und zu selbst daran erinnern muss, dass hier die Corona-Situation der Stein des Anstoßes war.

Neben den großen Fragen finden von Schirach und Kluge in den teilweise etwas mäandernden Ausführungen jedoch auch Zeit für persönliche Betrachtungen und Empfindungen sowie für die Erörterung ausgangsbeschränkt bedingter Missgeschicke im Haushalt.

Das Nachmittagsgespräch eröffnet sodann mit der Frage nach den Möglichkeiten der Literatur in Krisenzeiten und der programmatischen Erklärung: „Erzählen tröstet“ (von Schirach \& Kluge, 2020, S. 43). Doch die beiden Schriftsteller gehen durchaus weiter und verweisen bereits im vorangestellten Zitat auf Thomas Manns Der Tod in Venedig, dem auch der Titel des Bändchens entstammt: „Aschenbach hatte es einmal an wenig sichtbarer Stelle 


\section{Nordic Journal of Modern Language Methodology}

unmittelbar ausgesprochen, daß beinahe alles Große, was dastehe, als ein Trotzdem dastehe [...]“" (zitiert in von Schirach \& Kluge, 2020, S. 5).

Dies ist nicht nur ein Verweis auf die literarische Figur Aschenbach, die bei einer Cholera-Epidemie umkommt. Dieses Aschenbach'sche ,Trotzdem‘ scheint gerade für den zweiten Teil des Bändchens wegweisend zu sein, denn es spiegelt gleichsam die Kernannahme von Trotzdem wider: „,[D]as Virus hat uns an eine Zeitwende gebracht. Beides ist jetzt möglich, das Strahlende und das Schreckliche“"(von Schirach \& Kluge, 2020, S. 58). Ein Gemeinplatz sicherlich, aber in Trotzdem werden diese beiden zentralen Sätze, bildlich gesprochen, nicht einfach nur dahergesagt, sondern in einen literarischen und ideengeschichtlichen Klangkörper gestellt. In dieser Hinsicht erscheinen die Ausführungen zur Pest bzw. zu Boccaccio und Dante, zum Erdbeben von Lissabon sowie Kleists Das Erdbeben von Chili, sogar zu Zweigs Die Welt von Gestern und viele andere Verweise auf Denker und Dichter folgerichtig. Denn die aufgegriffenen Ereignisse bzw. ihre literarischen, ideengeschichtlichen und philosophischen Verarbeitungen scheinen in der Tat einem CoronaGrundgefühl verwandt: „Und jetzt plötzlich: Nichts stimmt mehr. Das, was wir für sicheren Grund hielten, ist weggebrochen“ (von Schirach \& Kluge, 2020, S. 57).

So ergeht denn auch die Warnung der geschichtsbewussten Juristen vor dem ,Schrecklichen', das vielen Deutschen, wenn auch in neuem, virulentem Gewand, unter Umständen erschreckend bekannt vorkommt:

Wir scheinen gerade zu erleben, dass es eine Art ungeschriebenen Notstand gibt und 95\% der Bevölkerung stimmen dem zu. Das ist gefährlich. Noch scheint unsere Demokratie nicht gefährdet, die Kanzlerin eine besonnene Frau, die Politiker sind in ihren Anstrengungen glaub- und vertrauenswürdig, die Maßnahmen sind zeitlich befristet. Aber es darf nicht zu lange dauern. Autoritäre Strukturen können sich verfestigen, die Menschen gewöhnen sich daran. Erosionen sind langsame Abtragungen, keine plötzlichen Ereignisse.

[...] Wir müssen um die Gefahren wissen, [...] wir müssen uns vor dem Ruf nach dem Leviathan hüten. (von Schirach \& Kluge, 2020, S. 70-72)

Aber nach allem Mahnen schließt das Buch dennoch mit der, zugegebenermaßen etwas vagen, Aussicht auf das ,Strahlende': 
Wir könnten heute über unsere Gesellschaft entscheiden - nicht wie sie ist, sondern so, wie wir sie uns wünschen. [...] Genau jetzt ist der richtige Zeitpunkt. Ist das nicht die eigentliche Aufgabe unserer Zeit? Würden wir damit nicht - nach der Barbarei des letzten Jahrhunderts - späteren Generationen etwas Strahlendes und Glückliches hinterlassen? Wären das [...] nach dem Gang durch die Hölle, nicht Dantes Sterne? (von Schirach \& Kluge, 2020, S. 74-75)

\section{Fazit für DaF-Lehrer}

Eine Wunderwaffe oder Allheilheilmittel zur besseren Vermittlung der Konjunktionen des Deutschen ist Trotzdem also nicht. Auch dürften die meisten DaF-Schüler mit der Lektüre dieses Bändchens überfordert sein, wenn auch nicht notwendigerweise aus sprachlichen Gründen, da sich die sprachliche Komplexität in Grenzen hält. Das liegt vermutlich am Format des Instant-Messanger-Gesprächs. Und nicht zuletzt können der Mansplaining-Ton sowie eine Reihe von Ungenauigkeiten das Lesevergnügen ein wenig trüben, wenn es dem jeweiligen Leser schwer fällt, die Prämissen dieses Bändchens einfach als gegeben anzunehmen.

Trotzdem - für den interessierten DaF-Lehrer sollte die Lektüre dieses Buches von Interesse sein, und dies gleich in mehrfacher Hinsicht. Zwar lassen sich die Reaktionen und Ausführungen von Schirachs und Kluges nicht mit einer universellen Reaktion in Deutschland gleichsetzen, doch verwundert weder das Erscheinen von Trotzdem noch die Resonanz in Form der Verkaufszahlen. Weiterhin gab Trotzdem dem Rezensenten bei der Behandlung des Scheiterns der Weimarer Republik und Verfassung sowie dem sog. Ermächtigungsgesetz, mit den norwegischen Germanistikstudenten Anlass für mehrere Abund Ausschweifungen zu aktuellen bundesdeutschen Debatten zu Notstandsmaßnahmen. Auch könnte eine Collage von relevanten Auszügen aus Trotzdem ein hilfreiches Instrument für DaF-Lehrer sein, um die landeskundliche Auseinandersetzung mit dem politischen System der BRD und der Stellung des Grundgesetzes zu unterstützen - oder auch kontrastiv zu beleuchten. Unmittelbar bzw. unaufbereitet verwenden lässt sich Trotzdem für den DaFUnterricht in Schulen in den meisten Fällen vermutlich nicht. Lesenswert ist das Buch allemal.

von Schirach, F., \& Kluge, A. (2020). Trotzdem. München: Luchterhand. 